\title{
Magnetostrophic MRI in the Earth's Outer Core
}

\author{
Ludovic Petitdemange, ${ }^{1,2}$ Emmanuel Dormy, ${ }^{1,3}$ and Steven A. Balbus ${ }^{1,2}$
}

We show that a simple, modified version of the Magnetorotational Instability (MRI) can develop in the outer liquid core of the Earth, in the presence of a background shear. It requires either thermal wind, or a primary instability, such as convection, to drive a weak differential rotation within the core. The force balance in the Earth's core is very unlike classical astrophysical applications of the MRI (such as gaseous disks around stars). Here, the weak differential rotation in the Earth core yields an instability by its constructive interaction with the planet's much larger rotation rate. The resulting destabilising mechanism is just strong enough to counteract stabilizing resistive effects, and produce growth on geophysically interesting timescales. We give a simple physical explanation of the instability, and show that it relies on a force balance appropriate to the Earth's core, known as magnetostrophic balance.

\section{Introduction}

The Magnetorotational Instability (MRI) is important for differentially rotating astrophysical objects such as gaseous disks, because it forces a breakdown of laminar flow into turbulence, producing the enhanced dissipation and transfer of angular momentum necessary for accretion of matter onto the central massive object (Balbus and Hawley, 1991). Disk investigators began with purely hydrodynamical candidate mechanisms, but now the center of interest is squarely upon magnetohydrodynamics. Interest in the Earth's core, by contrast, has been magnetic almost from the start. The principal problem, of course, has been to understand how fluid motions in the core generate a magnetic field. This is often approached via kinematic processes in which a hydrodynamically turbulent fluid exponentially amplifies a very weak seed magnetic field. Here, we argue of a version of the MRI, dynamically coupling both the velocity and the magnetic field, can also provide a mechanism for linear instability in the Earth's core. While this mechanism is not meant to serve as the primary dynamo process, it could be an important source of secondary instabilities and magnetic secular variation.

\footnotetext{
${ }^{1} \mathrm{MAG}(\mathrm{ENS} / \mathrm{IPGP})$

LRA, Département de Physique

Ecole Normale Supérieure

24, rue Lhomond

75231 Paris Cedex 05, France.

${ }^{2}$ LERMA, Observatoire de Paris, CNRS/UMR8112.

${ }^{3}$ Institut de Physique du Globe de Paris, CNRS/UMR7154.
} 
We are hardly the first authors to study the effects of differential rotation on the dynamical stability of the geodynamo (see e.g. Acheson, 1983; Ogden and Fearn, 1995; Fearn et al. 1997). But in previous calculations the emphasis has been upon purely azimuthal fields, nonaxisymmetric disturbances, and magnetic instabilities. In this work, the dynamical focus is much different. Here the magnetic coupling is to the poloidal field components, axisymmetric disturbances are front and center, and the instability, while relying on the presence of a magnetic field, has its seat of free energy entirely in differential rotation. The MRI is a somewhat novel concept in this context, and is worthy of study in isolation. Fortunately, it can be understood in very direct and simple physical terms.

The Earth's core is, by comparison to accretion disks, a relatively small object, in which resistive effects are on an equal footing with dynamical processes. The rotation properties of the Earth also significantly differ from those of an accretion disk. To leading order, they correspond to solid body rotation, with only a weak differential rotation. At first sight, it is far from obvious that the Earth's core is a venue for the MRI.

The purpose of this Letter is to show that, despite its weak differential rotation and significant resistive effects, the Earth's core can in fact host the MRI. We derive a WKB dispersion relation relevant to this asymptotic regime (i.e. magnetostrophic balance), and demonstrate excellent agreement between this local description and global numerical simulations in spherical geometry. The final section speculates on potential applications of our results.

\section{Modelling}

The physical parameter regime relevant to the Earth's outer core dynamics is relatively well constrained. In order to investigate the MRI in a conducting fluid, it is also necessary to provide a reasonable approximation to the basis flow profile over which the instability may develop. Apart from very restricted components of the velocity field, imaging of the core flow is limited to surface flows (e.g. Bloxham \& Jackson 1991; Amit \& Olson, 2004; Eymin \& Hulot, 2005). Moreover, while secular magnetic field variations allow for an order-of-magnitude estimate for the zonal flow (Pais and Hulot 2000), it is not possible at present to achieve a direct, detailed or reconstructive imaging of the global differential rotation of the Earth's core. Asymptotic studies early identified the essential role of the zonal shear in the geodynamo (Taylor, 1963), and this has remained central to our understanding of outer core dynamics. Numerical models of convection in rotating spheres (e.g. Christensen, 2002) or in convectively driven dynamos (e.g. Aubert, 2004), also observed the presence of a strong zonal shear. Besides surface flow reconstructions, the only (indirect) observational evidence for such shear is inferred from seismological data that have been interpreted as rotation of the solid inner core at a rate of about $0.15^{\circ}$ per year relative to the mantle (Vidale, Dodge \& Earle, 2000; see also Dumberry 2007). But is this change in the angular velocity profile spread across the whole core radius or, say, localised in a narrow shear layer? There are no observational constraints on the way the corresponding jump in angular velocity is actually accommodated by the flow. 


\section{Magnetostrophic-MRI local description}

Consider the stability of a differentially rotating fluid, with angular velocity $\Omega$ a function of $s$ and $z$ in a standard cylindrical coordinate system $(s, \phi, z)$. The density $\rho$, pressure $P$, magnetic field $\boldsymbol{B}$ are functions of $s$ and $z$ in the equilibrium state, and of course depend on time $t$ as well when the equilibrium is perturbed. The fluid is characterized by a kinematic viscosity $\nu$ and resistivity $\eta$. We work in the WKB limit, assuming that all linearized perturbations have the space-time dependence

$$
Q \propto \exp \left(i k_{s} s+i k_{z} z+\sigma t\right)
$$

where $Q$ is an infinitesimal (Eulerian) disturbance in a fluid quantity. Our starting point is the general dispersion relation of a constant density fluid adapted from Menou, Balbus, \& Spruit (2004):

$$
\frac{k^{2}}{k_{z}^{2}} \widetilde{\sigma}_{\eta \nu}^{4}-\widetilde{\sigma}_{\eta \eta}^{2}\left[\frac{1}{s^{3}} \mathcal{D}\left(s^{4} \Omega^{2}\right)\right]-4 \Omega^{2}\left(\boldsymbol{k} \cdot \boldsymbol{V}_{\boldsymbol{A}}\right)^{2}=0
$$

where

$$
\mathcal{D}=\left(\frac{k_{s}}{k_{z}} \frac{\partial}{\partial z}-\frac{\partial}{\partial s}\right)
$$

and

$$
\begin{gathered}
\tilde{\sigma}_{\eta \nu}^{2}=\left[\left(\sigma+\eta k^{2}\right)\left(\sigma+\nu k^{2}\right)+\left(\boldsymbol{k} \cdot \boldsymbol{V}_{\boldsymbol{A}}\right)^{2}\right], \\
\tilde{\sigma}_{\eta \eta}^{2}=\left[\left(\sigma+\eta k^{2}\right)^{2}+\left(\boldsymbol{k} \cdot \boldsymbol{V}_{\boldsymbol{A}}\right)^{2}\right] .
\end{gathered}
$$

The Alfvén velocity $V_{A}$ is defined as

$$
\boldsymbol{V}_{\boldsymbol{A}}=\frac{\boldsymbol{B}}{\sqrt{\mu \rho}}
$$

where $\mu$ is the magnetic permitivity. Finally, we note that $\Omega$ is the full angular velocity, i.e. if $\Omega_{0}$ denotes the Earth rotation rate, and $\boldsymbol{V}$ the velocity in the rotating frame, then $\Omega=\Omega_{0}+V_{\phi} / s$.

To see whether the unstable MRI modes can be present in a very simple model, we consider the case of an initial poloidal magnetic field which is locally vertical and $\Omega$ is a function only of $s$. (We stress here that we use a vertical magnetic field for the sake of simplicity of the presentation, but that the overall conclusion can be easily extended to the case of more general applied fields.) Then

$$
\mathcal{D}\left(s^{4} \Omega^{2}\right)=-\left(4 \Omega^{2}+s \frac{d \Omega^{2}}{d s}\right) \equiv-\kappa^{2} .
$$

For the current application, the shear gradient is very small compared with $4 \Omega^{2}$, and $\kappa$, which is known as the "epicyclic frequency" in the astrophysical literature, is very nearly $2 \Omega$. With these assumptions, the dispersion relation becomes

$$
\frac{k^{2}}{k_{z}^{2}}\left[\left(\sigma+\eta k^{2}\right)\left(\sigma+\nu k^{2}\right)+\left(\boldsymbol{k} \cdot \boldsymbol{V}_{\boldsymbol{A}}\right)^{2}\right]^{2}+4 \Omega^{2}\left(\sigma+\eta k^{2}\right)^{2}+\left(\boldsymbol{k} \cdot \boldsymbol{V}_{\boldsymbol{A}}\right)^{2} s \frac{d \Omega^{2}}{d s}=0 .
$$

This is the form that we have used (see below) for comparison with numerical simulations.

In geodynamo applications, the viscosity is very small compared with the resistivity (the ratio is $10^{-5}-10^{-6}$ ) and it may be dropped. The instability of interest arises 
from the final term of this dispersion relation, if the angular velocity decreases outwards. This is a small term in the sense that $\Omega$ is regarded as large, and we expect that the growth rate $\sigma$ will itself be very small compared with $\Omega$. We may therefore drop all $\sigma$ terms from our dispersion relation, except for those which are multiplied by $\Omega^{2}$. (This procedure may be formalized by normalizing $\sigma$ with respect to $|s d \Omega / d s|$ and then treating

$$
\varepsilon \equiv \frac{s}{\Omega} \frac{d \Omega}{d s} \sim 10^{-6}
$$

as a vanishingly small expansion parameter.) We obtain

$$
\frac{k^{2}}{k_{z}^{2}}\left(\boldsymbol{k} \cdot \boldsymbol{V}_{\boldsymbol{A}}\right)^{4}+4 \Omega^{2}\left(\sigma+\eta k^{2}\right)^{2}+\left(\boldsymbol{k} \cdot \boldsymbol{V}_{\boldsymbol{A}}\right)^{2} s \frac{d \Omega^{2}}{d s}=0(9)
$$

This relatively simple dispersion relation has a correspondingly simple physical interpretation. Consider magnetostrophic balance with an axial wave number $\left(\boldsymbol{k}=k \boldsymbol{e}_{\boldsymbol{z}}\right)$,

$$
2 \boldsymbol{\Omega} \times \boldsymbol{V}=-\nabla \Pi+\frac{1}{\mu \rho}(\boldsymbol{B} \cdot \boldsymbol{\nabla}) \boldsymbol{B},
$$

where $\Pi$ includes the material and magnetic pressures as well as the centrifugal potential, along with the full induction equation

$$
\left(\frac{\partial}{\partial t}+\boldsymbol{V} \cdot \boldsymbol{\nabla}\right) \boldsymbol{B}=(\boldsymbol{B} \cdot \boldsymbol{\nabla}) \boldsymbol{V}+\eta \nabla^{2} \boldsymbol{B} .
$$

The equilibrium profile is to leading order $V_{\phi}=s\left[\Omega_{0}+\right.$ $\left.\left(s-s_{0}\right) d \Omega / d s\right]$, and $\boldsymbol{B}=B \boldsymbol{e}_{\boldsymbol{z}}$. Consider linear velocity perturbations $v_{s}$ and $v_{\phi}$, and magnetic field perturbations $b_{s}$ and $b_{\phi}$ of the form $\exp (\sigma t+i k z)$. In magnetostrophic balance, the radial and azimuthal linearized equations are respectively

$$
\begin{gathered}
-2 \Omega v_{\phi}=\frac{i k B_{0}}{\mu_{0} \rho} b_{s}, \\
2 \Omega v_{s}=\frac{i k B_{0}}{\mu_{0} \rho} b_{\phi} .
\end{gathered}
$$

The same components of the induction equation are

$$
\begin{gathered}
\left(\sigma+\eta k^{2}\right) b_{s}=i k B_{0} v_{s}, \\
\left(\sigma+\eta k^{2}\right) b_{\phi}=i k B_{0} v_{\phi}+s \frac{d \Omega}{d s} b_{s} .
\end{gathered}
$$

Combining (14) and (13) leads to

$$
\left(\sigma+\eta k^{2}\right) b_{s}=-\frac{1}{2 \Omega}\left(k V_{A}\right)^{2} b_{\phi}
$$

whereas (15) and (12) imply

$$
\left(\sigma+\eta k^{2}\right) b_{\phi}=\left[\frac{\left(k V_{A}\right)^{2}}{2 \Omega}+s \frac{d \Omega}{d s}\right] b_{s} .
$$

Notice the absence of pressure-like perturbations for axial wavenumbers. The last two equations combine to give

$$
\left(k V_{A}\right)^{4}+4 \Omega^{2}\left(\sigma+\eta k^{2}\right)^{2}+\left(k V_{A}\right)^{2} s \frac{d \Omega^{2}}{d s}=0,
$$

which is just equation (9) for axial wavenumbers.

By restricting the perturbations exclusively to the 
magnetic field components, equations (16) and (17) produce a clear picture of the instability with no "out-ofphase" terms. Consider an outward radial displacement and its associated radial magnetic field. If $k V_{A}$ is not too large, the radial field is significantly sheared by the differential rotation to produce a negative azimuthal elongation of the field line following equation (17). This results in a positive azimuthal magnetic tension force, which must be balanced in the magnetostrophic regime with an opposing Coriolis force, hence with a yet greater radially outward displacement and magnetic field (16). An instability is at hand. This mechanism is illustrated in Figure 1, and seen through a direct simulation in Figure 2 .

The growing solution for $\sigma$ is

$$
\sigma=\frac{\left|\boldsymbol{k} \cdot \boldsymbol{V}_{\boldsymbol{A}}\right|}{2 \Omega}\left[\left|s \frac{d \Omega^{2}}{d s}\right|-\frac{k^{2}}{k_{z}^{2}}\left(\boldsymbol{k} \cdot \boldsymbol{V}_{\boldsymbol{A}}\right)^{2}\right]^{1 / 2}-\eta k^{2} .
$$

We wish to find the maximum growth rate of the instability, which will be associated with a particularly wave vector $\boldsymbol{k}$. The easiest way to proceed is to use the variables

$$
X=k \cdot \boldsymbol{V}_{\boldsymbol{A}}, \quad Y=\frac{k^{2}}{k_{z}^{2}},
$$

with the understanding that $X>0$, and the parameters

$$
\Lambda=\frac{V_{A}^{2}}{2 \eta \Omega_{0}}=\frac{B_{0}^{2}}{2 \mu_{0} \rho \eta \Omega_{0}}, \quad a=\left|s \frac{d \Omega^{2}}{d s}\right|
$$

$\Lambda$ is the Elsasser number, which is typically of order unity for the Earth's core. Then

$$
2 \Omega \sigma=X\left(a-X^{2} Y\right)^{1 / 2}-X^{2} Y / \Lambda .
$$

This is a decreasing function of $Y$ everywhere, with a maximum at the lower boundary $Y=1$. This means that the most rapidly growing wavenumber $k_{z}$ lies along the rotation axis. Thus

$$
2 \Omega \sigma_{(\max )}=X\left(a-X^{2}\right)^{1 / 2}-X^{2} / \Lambda .
$$

Requiring the partial derivative $\partial / \partial X$ to vanish leads to the polynomial

$$
X^{4}-a X^{2}+\frac{a^{2}}{4\left(1+\Lambda^{-2}\right)}=0 .
$$

The physically meaningful wavenumber solution is

$$
X^{2} \equiv\left(k_{z} V_{A}\right)^{2}=\Omega\left|s \frac{d \Omega}{d s}\right|\left[1-\left(1+\Lambda^{2}\right)^{-1 / 2}\right],
$$

and corresponds to a growth rate of

$$
\sigma=\left|s \frac{d \Omega}{d s}\right| \frac{\Lambda / 2}{1+\sqrt{1+\Lambda^{2}}} .
$$

It is of interest to examine the range of $k_{z}$ in which instability exists. For the case $k=k_{z}$, the dispersion relation (9) admits growing solutions for values of $k_{z}^{2}$ less than

$$
k_{z}^{2}=\frac{\left|s d \Omega^{2} / d s\right|}{V_{A}^{2}+4 \Omega^{2} \eta^{2} / V_{A}^{2}} .
$$


Notice that when $V_{A}$ is very large, $k_{z}$ is very small because large $k_{z}$ perturbations are stabilized by magnetic tension forces. Conversely, when $V_{A}$ is tiny, resistivity damps large wavenumber perturbations. The maximum value of $k_{z}$ allowing the greatest range of unstable wavenumbers corresponds to $\Lambda=1$. Hence, Elsasser numbers of order unity naturally emerge in an MRIinfluenced dynamo.

At this point it is helpful to have some explicit numbers. We take $\Omega=7.27 \times 10^{-5} \mathrm{~s}^{-1}$ and $s d \Omega / d s \simeq 10^{-10}$ $\mathrm{s}^{-1}$, the latter corresponding to a lower bound for the angular velocity gradient in which the characteristic length scale associated with shear in the core is the outer core radius itself (worse case MRI scenario). With $\Lambda=1$ and $\eta \simeq 1 \mathrm{~m}^{2} \mathrm{~s}^{-1}$, the Alfvén velocity is $0.012 \mathrm{~m} \mathrm{~s}^{-1}$. From equation (25), we find

$$
k V_{A} \simeq 4.6 \times 10^{-8} \mathrm{~s}^{-1}
$$

which translates to a wavelength of some $1600 \mathrm{~km}$. The characteristic growth time from equation (26) is then about 1500 years. If, on the other hand, we assume that a similar jump in angular velocity is accomodated accross a narrow shear layer (such as the Stewartson layer, Stewartson 1966), then the characteristic growthtime could be as short as a year.

What is the relationship between the unstable mode considered here and the maintenance of the classical Taylor constraint? It is well known that in the magnetostrophic limit, the geostrophic component of the flow must adjust to ensure Taylor's constraint (Taylor, 1964). A modification of the non-geostrophic flow similar to the one envisioned here could in principle rapidly alter the zonal shear on which the instability itself relies. However, in the particular configuration investigated, the wavenumber is axial. Thus, at least in the linear phase of the disturbance, the Taylor constraint remains unaffected.

\section{Direct numerical simulations}

To demonstrate numerically the above mechanism, we base our study on a very idealized model of the Earth core, chosen for illustrative purposes. We consider simple spherical Couette flow driven by enforcing differential rotation between the inner core and the mantle. The applied magnetic field $B_{0}$ is vertical and uniform. In the simpler hydrodynamical case, a strong shear layer will develop on the cylinder tangent to the inner core (Proudman, 1956; Stewartson, 1966). With the particular choice of a vertical applied field, this shear is only slightly modified by MHD effects (see however Dormy et al., 1998). In what follows, we will work in time units of $\left(2 \Omega_{0}\right)^{-1}$ and space units of $r_{o}$, the radius of the outer sphere. It is also convenient to introduce the dimensionless velocity

$$
\boldsymbol{U}_{\mathbf{0}}=\frac{\boldsymbol{V}_{\mathbf{0}}}{2 r_{o} \Omega_{0}}
$$

where $\boldsymbol{V}_{\mathbf{0}}$ is the unperturbed velocity measured in the frame rotating at $\Omega_{0}$. As before, the linear perturbed velocity is $\boldsymbol{v}$. For the perturbed velocity $\boldsymbol{v}$ and magnetic field $\boldsymbol{b}$, we introduce dimensionless variables $\boldsymbol{v}^{\prime}$ and $\boldsymbol{b}^{\prime}$ :

$$
\boldsymbol{v}^{\prime}=\boldsymbol{v} /\left(2 r_{o} \Omega_{0}\right), \quad \boldsymbol{b}^{\prime}=\boldsymbol{b} / B_{0} .
$$


The governing fluid equations may then be written

$\frac{\partial \boldsymbol{v}^{\prime}}{\partial t}+\left(\boldsymbol{U}_{\mathbf{0}} \cdot \boldsymbol{\nabla}\right) \boldsymbol{v}^{\prime}+\left(\boldsymbol{v}^{\prime} \cdot \nabla\right) \boldsymbol{U}_{\mathbf{0}}=-\nabla \pi+E \Delta \boldsymbol{v}^{\prime}+\frac{E \Lambda}{P m}\left(\nabla \times \boldsymbol{b}^{\prime}\right) \times \boldsymbol{e}_{\boldsymbol{z}}-\boldsymbol{e}_{z} \times \boldsymbol{v}^{\prime}$

$$
\begin{gathered}
\frac{\partial \boldsymbol{b}^{\prime}}{\partial t}=\boldsymbol{\nabla} \times\left(\boldsymbol{v}^{\prime} \times \boldsymbol{e}_{\boldsymbol{z}}+\boldsymbol{U}_{\mathbf{0}} \times \boldsymbol{b}^{\prime}\right)+\frac{E}{P m} \Delta \boldsymbol{b}^{\prime}, \\
\boldsymbol{\nabla} \cdot \boldsymbol{v}^{\prime}=\boldsymbol{\nabla} \cdot \boldsymbol{b}^{\prime}=0 .
\end{gathered}
$$

In addition to the Elsasser number $\Lambda$, we have introduced the standard Ekman and magnetic Prandtl numbers, which are respectively:

$$
E=\nu /\left(2 \Omega_{0} r_{o}^{2}\right) \quad P m=\nu / \eta,
$$

and $\pi$ is the perturbed value of the dimesionless pressure.

As a model for the Earth's core, we investigate a spherical shell. The angular velocity $\Omega_{0}$ is defined by the outer sphere. In this reference frame, the inner sphere rotates with angular velocity $\Omega_{i}>0$, in order for the differential rotation to decrease outward. We define the Rossby number as

$$
R o=\Omega_{i} / \Omega_{0}
$$

Let us now compare the local WKB analysis (8) with the global growth rate obtained numerically. We first need to compute the dimensionless steady velocity profile $\boldsymbol{U}_{\mathbf{0}}$. If the magnetic field is weak enough, this can be obtained as a solution of the hydrodynamic problem. The results are displayed in Table 1. If the Elsasser number (21) becomes large, the field obviously affects the steady solution, and we then perform simulations with the steady MHD state as initial configuration, see Table 2 .

To best illustrate the above theoretical analysis, we use numerical values as close as feasible to the regime described. Simulations use $P m=0.5$, the differential rotation is weak compared to the planet rotation $(R o<<1)$, and the Ekman number is decreased to small values. To accommodate small Ekman numbers, we have implemented an axisymetric version of the Parody code (Dormy et al., 1998, Christensen et al., 2001, and later collaborative developments) with radial resolution ranging from 500 to 1000 points in radius and between 200 and 500 harmonics.

Our results are summarized in Table 1. In agreement with theoretical expectations, the radial wavevector $k_{s}$ varies approximately in proportion to the dominant shear, i.e. $E^{1 / 4}$. The value of $k_{s}$ is taken from numerical simulations by computing the width of the unstable mode at mid-intensity, and the value of $k_{z}$ is obtained by direct fourier transform. It is found that the most unstable mode's half wavelength generally occupies the full radial extent of the sheared region. The inner core and the associated equatorial singularity of the Ekman layer are the natural modal boundaries; the equatorial singularity divides the tangent cylinder into independent domains. Finally, to limit the stabilizing effect of diffusion, we must use $E$ much less than unity.

The induced azimuthal magnetic field clearly reproduces the mechanism of the magnetostrophic MRI (see figure 1), despite the presence of finite inertial and diffusive effects (which were neglected in the theoretical calculation), and the complications associated with a bounded spherical domain (also neglected).

Some of these cases involve large values of the Elsasser 
number, one may then worry that they are not fully self consistent. To check this, we show, in Table 2, the instability parameters of such strong field profiles, which now also have a (weak) dependence on $\Lambda$. For the field geometry used here, however, both the rotation profile and the applied field require relatively little adjustment. Agreement with the local description is also obtained for such configurations.

\section{Conclusions and Discussion}

At the very least, the magnetostrophic MRI discussed here seems to be an efficient regulatory mechanism in the outer core, providing a back reaction against the build up of angular velocity at smaller radii. This could be tested in full scale numerical simulations of spherical shells whose gross aspect and parameters could be regulated either to admit the MRI or not.

More interestingly, might there be traces of the magnetostrophic MRI in geomagnetic observations? Because of the severe limitations in our knowledge of differential rotation within the Earth's core, it is not possible to offer any kind of definitive treatment. It is, however, interesting to note that some axial variation in the zonal flow is required to account for changes in the length of the a day over millenial timescales (Dumberry \& Bloxham 2006). In that respect It is interesting that axial wavenumber disturbances grow particularly rapidly in the model studied here.

Another temptative application is based on the observation that the growth rate in question is directly proportional to the shear, which could be highly localized. Thus, growth times considerably shorter than 1500 years are possible: in principle, shear times of order years could be produced. This suggests a possible connection with the observations of rapid events of internal origin, known as "geomagnetic jerks" or "geomagnetic impulses". These rapid changes in the secular variation were detected and characterized from observatory data (Courtillot et al., 1978; Le Mouël et al., 1982) and found to correspond to localised patches of rapid field variation at the surface of the core, using global field models (Dormy and Mandea, 2005). We reemphasize, however, that the establishment of a compelling link between MRI unstable configurations in the Earth core and geomagnetic impulses will require considerably more effort. Nevertheless, the MRI offers for consideration a novel magnetohydrodynamic mechanism that is able to induce significant field variations over a range of observationally interesting timescales.

Acknowledgments. We thank M. Dumberry and an anonymous referee for constructive criticisms that greatly improved the presentation of the paper. Computations reported here were carried out on the CEMAG computing center at LRA/ENS. SAB acknowledges support from the French Ministry of Higher Education in the form of a Chaire d'Excellence award with additional funds from the Région Ile de France.

\section{References}

Acheson, D.J. (1983), Local analysis of thermal and magnetic instabilities in a rapidly rotating fluid, Geophys. Astrophys. Fluid Dynam., 27, 123-136

Amit, H. and P. Olson (2004), Helical core flow from geomagnetic secular variation, Phys. Earth Plan. Int., 147, 1-25.

Aubert, J. (2005), Steady zonal flows in spherical shell dynamos, J. Fluid Mech., 542, 53-67. 
Balbus, S.A. and J.F. Hawley (1991) A powerful local shear instability in weakly magnetized disks, Astrophys. J., 376, $214-222$.

Bloxham, J., and A. Jackson (1991), Fluid flow near the surface of earth's outer core, Rev. Geophys., 29, 97-120.

Christensen, U. et al (2001) A numerical dynamo benchmark, Phys. Earth Plan. Int., 128, 25-34.

Christensen, U. (2002), Zonal flow driven by strongly supercritical convection in rotating spherical shells, J. Fluid Mech., 470, 115-133.

Courtillot, V., J. Ducruix, J.L. Le Mouël (1978), Sur une accélération récente de la variation séculaire du champ magnétique terrestre, Acad. Sci., Paris, C.R., D287, 10951098.

Dormy, E., P. Cardin and D. Jault (1998), MHD flow in a slightly differentially rotating spherical shell, with conducting inner core, in a dipolar magnetic field, Earth and Planetary Science Letters, 160, 15-30.

Dumberry, M. (2007), Geodynamics constraints on the steady and time-dependent inner core axial rotation, Geophys. J. Int., 170, 886-895.

Dumberry, M. and J. Bloxham (2006), Azimuthal flows in the Earth's core and changes in the length of the day at millenial time scales, Geophys. J. Int., 165, 32-46.

Dormy, E. and M. Mandea (2005), Tracking geomagnetic impulses at the core-mantle boundary, Earth Planet. Sci. Lett., 237, 300-309.

Eymin, C. and G. Hulot (2005), On core surface flows inferred from satellite magnetic data, Phys. Earth Plan. Int., 152, 200-220.

Fearn, D.R., C.J. Lamb, D.R. McLean,R.R. Ogden (1997), The influence of differential rotation on magnetic instability, and nonlinear magnetic instability in the magnetostrophic limit, Geophys. Astrophys. Fluid Dynam., 86, $173-200$.

Le Mouël, J.L., J. Ducruix, C. Ha Duyen (1982), The worldwide character of the 1969-1970 impulse of the secular acceleration rate, Phys. Earth Planet. Inter., 28, 337-350.

Menou K., S.A. Balbus, and H.C. Spruit (2004), Local axisymmetric diffusive stability of weakly magnetized, differentially rotating, stratified fluids, Astrophys. J., 607, 564574.

Ogden, R.R., D.R. Fearn (1995), The destabilising role of differential rotation, Geophys. Astrophys. Fluid Dynam., 81, 215-232.

Proudman, I. (1956), The almost-rigid rotation of viscous fluid between concentric spheres, J. Fluid Mech., 1, 505-516.

Stewartson, K. (1966), On almost rigid rotations, Part 2, J. Fluid Mech., 26, 131-144.

Taylor, J.B. (1964), The Magneto-Hydrodynamics of a Rotating Fluid and the Earth's Dynamo Problem, Proc. Roy. Soc. Lond., 274, 274-283.

Vidale, J. E., D. A. Dodge, and P.S. Earle (2000), Slow differential rotation of the Earth's inner core indicated by temporal changes in scattering, Nature, 405, 445-448.

Ludovic Petitdemange, Emmanuel Dormy, Steven Balbus, LRA, Département de Physique, Ecole Normale Supérieure, 24, rue Lhomond, 75231 Paris Cedex 05, France (dormy@phys.ens.fr) 
X -10

PETITDEMANGE ET AL.: MRI IN THE EARTH'S OUTER CORE

Table 1. Results obtained for the most unstable mode in a direct integration of the governing equations. We list the growth rate $\left(\sigma_{\text {num }}\right)$ and the wavenumber $\left(k_{s}\right.$ and $\left.k_{z}\right)$. We also list the growth rate $\left(\sigma_{\mathrm{th}}\right)$ obtained directly from the local dispersion relation (8) with these wavenumber values. The variables $E, R o$, and $\Lambda$ are defined in equations (21), (33) and (34). The numerical agreement is excellent.

\begin{tabular}{lcccccc}
\hline $2 E$ & $R o$ & $2 \Lambda$ & $\sigma_{\text {th }}$ & $\sigma_{\text {num }}$ & $k_{s}$ & $k_{z}$ \\
\hline $2 \times 10^{-6}$ & 0.005 & 1.0 & $6 \times 10^{-4}$ & $6.4 \times 10^{-4}$ & $\pi / 0.17$ & $\pi / 0.25$ \\
$10^{-6}$ & & 25 & $1.0 \times 10^{-3}$ & $1.09 \times 10^{-3}$ & $\pi / 0.17$ & $\pi / 0.6$ \\
& 0.01 & 5 & $8.8 \times 10^{-3}$ & $1.16 \times 10^{-2}$ & $\pi / 0.14$ & $\pi / 0.15$ \\
& 0.01 & 25 & $1.0 \times 10^{-2}$ & $1.0 \times 10^{-2}$ & $\pi / 0.14$ & $\pi / 0.33$ \\
& 0.005 & 25 & $3.6 \times 10^{-3}$ & $3.6 \times 10^{-3}$ & $\pi / 0.14$ & $\pi / 0.4$ \\
& 0.005 & 5 & $4.2 \times 10^{-3}$ & $4.8 \times 10^{-3}$ & $\pi / 0.14$ & $\pi / 0.1$ \\
$5 \times 10^{-7}$ & 0.0025 & 25 & $2.0 \times 10^{-4}$ & $2.3 \times 10^{-4}$ & $\pi / 0.14$ & $\pi / 0.6$ \\
& 0.005 & 50 & $4.0 \times 10^{-3}$ & $4.4 \times 10^{-3}$ & $\pi / 0.11$ & $\pi / 0.25$ \\
& 0.005 & 25 & $6.0 \times 10^{-3}$ & $6.4 \times 10^{-3}$ & $\pi / 0.11$ & $\pi / 0.14$ \\
& & 15 & $7.0 \times 10^{-3}$ & $6.2 \times 10^{-3}$ & $\pi / 0.11$ & $\pi / 0.125$ \\
& & 10 & $7.1 \times 10^{-3}$ & $7.6 \times 10^{-3}$ & $\pi / 0.11$ & $\pi / 0.1$ \\
& & 5 & $6.0 \times 10^{-3}$ & $6.4 \times 10^{-3}$ & $\pi / 0.1$ & $\pi / 0.071$ \\
& & 2 & $3.0 \times 10^{-3}$ & $4.8 \times 10^{-3}$ & $\pi / 0.1$ & $\pi / 0.067$ \\
& & 1 & $2.24 \times 10^{-3}$ & $3.0 \times 10^{-3}$ & $\pi / 0.1$ & $\pi / 0.058$ \\
& 0.5 & $1.16 \times 10^{-3}$ & $1.36 \times 10^{-3}$ & $\pi / 0.11$ & $\pi / 0.11$ \\
& 0.005 & 0.3 & $6.96 \times 10^{-4}$ & $2.03 \times 10^{-4}$ & $\pi / 0.1$ & $\pi / 0.14$ \\
$2.5 \times 10^{-7}$ & 0.0025 & 25 & $2.0 \times 10^{-3}$ & $1.93 \times 10^{-3}$ & $\pi / 0.1$ & $\pi / 0.25$ \\
$10^{-7}$ & 0.0075 & 25 & $6.2 \times 10^{-3}$ & $6.6 \times 10^{-3}$ & $\pi / 0.1$ & $\pi / 0.2$ \\
& 0.0025 & 25 & $2.0 \times 10^{-3}$ & $3.2 \times 10^{-3}$ & $\pi / 0.08$ & $\pi / 0.15$ \\
& & 25 & $4.0 \times 10^{-4}$ & $2.2 \times 10^{-4}$ & $\pi / 0.06$ & $\pi / 0.04$ \\
& & & $4.0 \times 10^{-3}$ & $4.8 \times 10^{-3}$ & $\pi / 0.06$ & $\pi / 0.083$ \\
\hline
\end{tabular}

Table 2. Results obtained using the steady MHD state as initial configuration. Computations are performed with $R o=$ $5 \times 10^{-3}, P m=0.5$ and $E=2.5 \times 10^{-7}$. As for Table 1, both the numerical parameters of the most unstable mode and the corresponding theoretical growth rate are reported.

\begin{tabular}{lcccc}
\hline $2 \Lambda$ & $\sigma_{\text {th }}$ & $\sigma_{\text {num }}$ & $k_{r}$ & $k_{z}$ \\
\hline 3 & $5.06 \times 10^{-3}$ & $5.70 \times 10^{-3}$ & $\pi / 0.92$ & $\pi / 0.056$ \\
1 & $2.06 \times 10^{-3}$ & $1.84 \times 10^{-3}$ & $\pi / 0.95$ & $\pi / 0.056$ \\
0.3 & $8.44 \times 10^{-4}$ & $2.76 \times 10^{-4}$ & $\pi / 0.10$ & $\pi / 0.11$ \\
\hline
\end{tabular}


a.

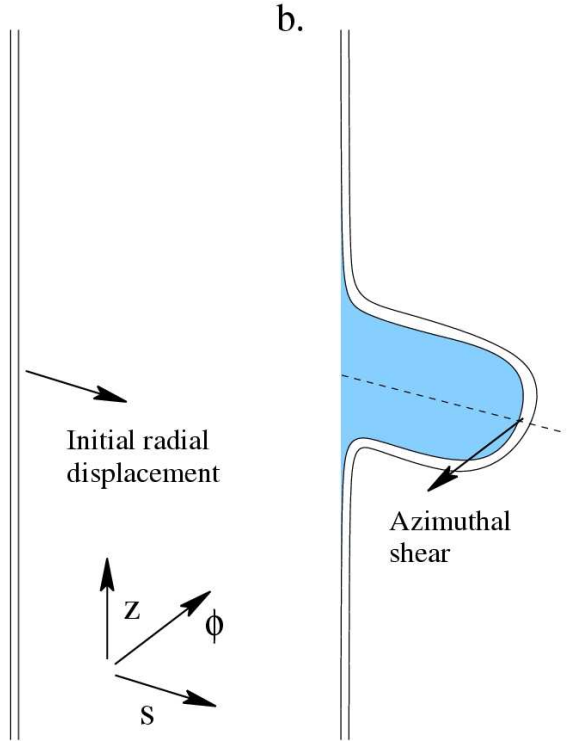

c.

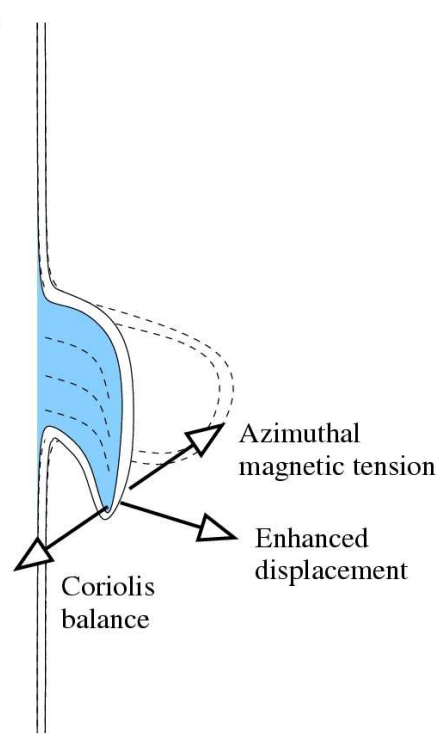

Figure 1. Development of the magnetostrophic MRI. (a) Unperturbed field line. (b) Field line is distorted by a radially outward displacement, and subject to velocity shear. (c) Field line develops azimuthal tension which is immediately compensated by the Coriolis force. This compensating force requires a further displacement in the same sense of the initial outward radial extension, and the instability proceeds.

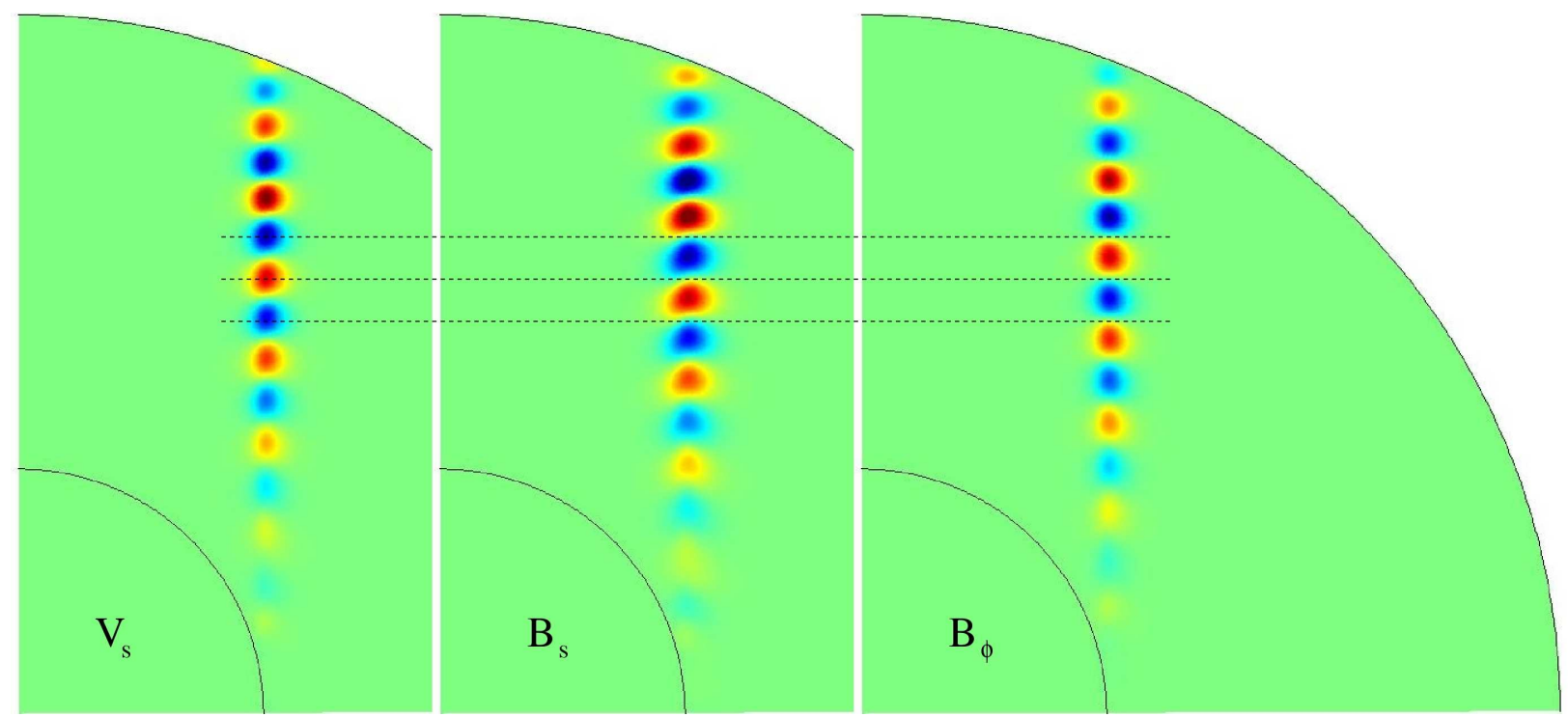

Figure 2. Snapshot of the most unstable mode in a direct simulation with $E=5 \times 10^{-7}, \Lambda=3$ and $P m=0.5$. The physical mechanism of the magnetostrophic-MRI can be traced in the phase shifts between $v_{s}, b_{s}$ and $b_{\phi}$ : $b_{s}$ is a quarter-period ahead of $v_{s}$, while $b_{\phi}$ is exactly out of phase by half a period with $b_{s}$. 